ESAIM: PROCEEDINGS AND SURVEYS, November 2014, Vol. 46, p. 47-62

ECIT 2012 - Witold Jarczyk, Daniele Fournier-Prunaret, João Manuel Gonçalves Cabral

\title{
RECENT RESULTS ON ITERATIVE ROOTS
}

\author{
WITOLD JARCZYK ${ }^{1}$
}

\begin{abstract}
This is a rough survey of some results on iterative roots (fractional iterates) published recently. Also some historical information to clear the connection to previous results has been given. The main topics are: the conjugacy of piecewise monotonic functions and their iterative roots, stability of iterative roots, some substitutes and generalizations of the notion of iterative root using set-valued functions.
\end{abstract}

Résumé. Le présent article de revue approximative contient de certains résultats sur les racines itératives (itérées fractionnaires) publiés récemment. Une information historique est aussi donnée pour montrer la liason avec des résultats précédents. Les sujets principaux sont: la conjugaison des fonctions monotones par morceaux et leurs racines itératives, stabilité des racines itératives, certains substituts et généralizations de la notion de la racine itérative qui utilisent les fonctions multivaluées.

\section{INTRODUCTION}

This is an extension of a survey talk presented during the European Conference on Iteration Theory, held in Ponta Delgada, Azores, Portugal, from September 9 to September 15, 2012. It gives a short progress report on iterative roots of self-mappings of a real interval. This is only a personal choice of papers and results published mainly in last several years.

We are interested in continuous iterative roots (or: fractional iterates) of a given continuous self-mapping $F$ of a closed interval $[a, b]$, where $a, b \in \mathbb{R}, a<b$. To be precise the problem is as follows. Let $n \geq 2$ be an integer. Find a continuous $f:[a, b] \rightarrow[a, b]$ such that the $n$-th iterate $f^{n}$ of $f$, i.e. the composition of $n$ copies of $f$, is $F$ :

$$
f^{n}(x)=F(x)
$$

for each $x \in[a, b]$. It seems that it was Ch. Babbage who first, yet at the beginning of the 19th century, wrote on iterative roots explicitly. In [1] he studied (0.1) for $F$ being the identity mapping (see also [46, Sct. 1.7 and 3.7]). After him a great number of results, concerning the general case of (0.1) in various settings, has been proved. Many of them can be found in the monographs [18] and [19] by M. Kuczma and M. Kuczma, B. Choczewski, R. Ger, respectively, as well as in the book [58] and article [60] by Gy. Targonski. For some

1 Faculty of Mathematics, Computer Science and Econometrics, University of Zielona Góra, Szafrana 4a, PL-65-516 Zielona Góra, Poland, e-mail: : w.jarczyk@wmie.uz.zgora.pl

Mathematics Subject Classification (2013). Primary: 3902, 39B12, 26A18; Secondary: 26A48, 54C60.

Keywords. iteration, iterative root, topological conjugacy, stability, piecewise monotonic function, set-valued function, formal power series, homeomorphism of the circle

Mots clefs. itération, racine itérative, conjugaison topologique, stabilité, fonction monotone á morceaux, fonction

multivaluée, série formelle, homéomorphisme du cercle

(c) EDP Sciences, SMAI 2014 
other results, published after 1990, the reader is referred to the article [69] by Jingzhong Zhang, Lu Yang and Weinian Zhang, to the survey papers [3] and [2], and the recent survey article [66] by M.C. Zdun and P. Solarz.

This paper deals with the following five topics which simultaneously are the titles of consecutive sections:

1. Piecewise monotonic functions and their conjugacy

2. Iterative roots of piecewise monotonic functions

3. Stability problems for iterative roots. Aproximate roots

4. Multifunction case

5. Not discussed although important

Section 4 concerns with some possible generalizations of the notion of iterative root.

\section{PieceWise monotonic FUnCtions And THEIR CONJUGACY}

A point $t$ of the interval $(a, b)$ is called a turning point of $F$ if $F$ has a local extremum at $t$. The function $F$ is said to be $r$-modal, $r \in \mathbb{N}_{0}$, if it has exactly $r$ turning points, and piecewise monotonic if it has only finitely many of them. By $\mathcal{M}_{r}(a, b)$ we denote the set of all $r$-modal self-mappings of $[a, b]$, whereas $\mathcal{P} \mathcal{M}(a, b)$ will denote the set of all piecewise monotonic functions mapping $[a, b]$ into itself. If $F \in \mathcal{P M}(a, b)$ then we denote by $S(F)$ the set of all turning points of $F$. Observe that

$$
\mathcal{P M}(a, b)=\bigcup_{r=0}^{\infty} \mathcal{M}_{r}(a, b) .
$$

Of course, in case when $S(F)=\left\{t_{1}, \ldots, t_{r}\right\}$ and $a=t_{0}<t_{1}<\ldots<t_{r}<t_{r+1}=b$ for some $r \in \mathbb{N}$, then $\left.F\right|_{\left[t_{i}, t_{i+1}\right]}$ is strictly monotonic for each $i \in\{0, \ldots, r\}$. The following simple facts can be found in [26].

Fact 1.1. Let $F \in \mathcal{P} \mathcal{M}(a, b)$. Then $S\left(F^{k}\right) \subset S\left(F^{k+1}\right)$ for every $k \in \mathbb{N}$.

If there is a $k \in \mathbb{N}$ such that $S\left(F^{k}\right)=S\left(F^{k+1}\right)$, then the least one is called the nonmonotonicity height of $F$ and denoted by $H(F)$. Otherwise we put $H(F)=\infty$.

Fact 1.2. Let $F \in \mathcal{P} \mathcal{M}(a, b)$. Then $S\left(F^{k}\right)=S\left(F^{H(F)}\right)$ for every integer $k \geq H(F)$.

It is a good idea to classify piecewise monotonic functions with respect to their heights.

Fact 1.3. Let $F \in \mathcal{P} \mathcal{M}(a, b)$ and $k \in \mathbb{N} \cup\{0\}$. Then $H(F) \leq k$ if and only if $F$ is strictly monotonic on $F^{k}([a, b])$. In particular, $H(F)=0$ if and only if $F$ is strictly monotonic.

One can observe that a lower height implies a simpler dynamic behaviour, and vice versa; in particular, the infinite height usually means a complicated, sometimes even chaotic in a sense, behaviour. For instance this is the case in the example below, where the considered function has cycles of all orders, so it is chaotic in the sense of $\mathrm{Li}$ and Yorke, and has a positive topological entropy.

Example 1.4. For the classical hat function $F:[0,1] \rightarrow[0,1]$, given by $F(x)=\min \{2 x, 2-2 x\}$, we have

$$
S\left(F^{k}\right)=\left\{\frac{1}{2^{k}}, \ldots, \frac{2^{k}-1}{2^{k}}\right\}, \quad k \in \mathbb{N},
$$

whence $H(F)=\infty$.

The notion of conjugacy is one of the most important in iteration theory. In particular, it is closely related to iterative roots. Continuous self-mappings $F$ and $G$ of intervals $[a, b]$ and $[c, d]$, respectively, are said to be (topologically) conjugate if there is a homeomorphism $\phi$ mapping $[a, b]$ onto $[c, d]$, satisfying the conjugacy equation

$$
\phi \circ F=G \circ \phi .
$$


A number of papers has been devoted to conjugacy of piecewise monotonic functions. Two recent papers providing some sufficient conditions for conjugacy of such functions should be definitely mentioned here. The first one, viz. [15], is due to Ch. Kawan who gave such conditions in the case of expanding piecewise monotonic functions. Expansivity of an $F \in \mathcal{P} \mathcal{M}(a, b)$ with $S(F)=\left\{t_{1}, \ldots, t_{r}\right\}$, where $a=t_{0}<t_{1}<\ldots<t_{r}<t_{r+1}=b$, means here that $F$ admits a metric $d$ in $[a, b]$, generating the Euclidean topology, and a number $\mu \in(0, \infty)$ such that $d(F(x), F(y)) \geq \mu d(x, y)$ for all $x, y \in\left[t_{i}, t_{i+1}\right]$ and $i \in\{0, \ldots, r\}$. A more general situation was studied in the paper [9] by K. Ciepliński and M. C. Zdun who examined the conjugacy in a larger class of so-called $\gamma$-expanding piecewise monotonic functions. The main tools in both papers are fixed point theorems. This is Banach's contraction principle in the paper [15], and a more subtle result, viz. Matkowski's fixed point theorem, in $[9]$.

Let me focus on the very fresh papers [43] and [44] by Yong-Guo Shi, Lin Li, and Zb. Leśniak. The main result of the first one provides a characterization of conjugacy in very special families $\mathcal{M}_{r,+}(a, b)$ and $\mathcal{M}_{r,-}(a, b)$ of piecewise monotonic functions.

Given a number $r \in \mathbb{N}$ define $\mathcal{M}_{r,+}(a, b)$ as the class of all functions $F$ from $\mathcal{M}_{r}(a, b)$ such that the following condition holds, where $S(F)=\left\{t_{1}, \ldots, t_{r}\right\}$ and $a<t_{1}<\ldots<t_{r}<b$ : $F(x)<x$ for each $x \in\left(a, t_{1}\right]$ and, for every $i \in\{1, \ldots, r\}$,

$$
\text { if } i \text { is even, then } F\left(t_{i}\right)=F(a)=a \text { and if } i \text { is odd, then } F\left(t_{i}\right)=F\left(t_{1}\right) \geq F(b) \text {. }
$$

On the other hand, the conditions $F(x)>x$ for each $x \in\left[t_{r}, b\right)$ and, for every $i \in\{1, \ldots, r\}$,

$$
\text { if } r-i \text { is even, then } F\left(t_{i}\right)=F\left(t_{r}\right) \leq F(a) \text { and if } r-i \text { is odd, then } F\left(t_{i}\right)=F(b)=b
$$

give the definition of the class $\mathcal{M}_{r,-}(a, b)$. Observe that if $F \in \mathcal{M}_{r,+}(a, b)$ for some $r \in \mathbb{N}$, then $F([a, b])=$ $\left[a, F\left(t_{1}\right)\right] \subset\left[a, t_{1}\right]$, and if $F \in \mathcal{M}_{r,-}(a, b)$ then $F([a, b])=\left[F\left(t_{r}\right), b\right] \subset\left[t_{r}, b\right]$. Consequently, since $F$ is not monotonic, we see that $H(F)=1$ in both cases.

The following result by Yong-Guo Shi, Lin Li and Zb. Leśniak originates in the paper [43] and characterizes the conjugacy in the classes mentioned above. Before its formulating notice that if $F \in \mathcal{M}_{r,+}(a, b)$ then

$$
F\left(\left[a, t_{1}\right]\right)=\left[a, F\left(t_{1}\right)\right]=\{a\} \cup \bigcup_{k=1}^{\infty}\left(F^{k+1}\left(t_{1}\right), F^{k}\left(t_{1}\right)\right]
$$

and if $F \in \mathcal{M}_{r,-}(a, b)$ then

$$
F\left(\left[t_{r}, b\right]\right)=\left[F\left(t_{r}\right), b\right]=\{b\} \cup \bigcup_{k=1}^{\infty}\left[F^{k}\left(t_{r}\right), F^{k+1}\left(t_{r}\right)\right)
$$

Theorem 1.5. Let $F \in \mathcal{M}_{r}(a, b)$ and $G \in \mathcal{M}_{r}(c, d)$ with $S(F)=\left\{t_{1}, \ldots, t_{r}\right\}$ and $S(G)=\left\{u_{1}, \ldots, u_{r}\right\}$, where $a, b, c, d \in \mathbb{R}, a<t_{1}<\ldots<t_{r}<b$ and $c<u_{1}<\ldots<u_{r}<d$.

Assume that $F \in \mathcal{M}_{r,+}(a, b)$ and $G \in \mathcal{M}_{r,+}(c, d)$. Then $F$ and $G$ are conjugate if and only if either

$$
F(b)=a \quad \text { and } \quad G(d)=c,
$$

or

or

$$
F(b)=F^{k}\left(t_{1}\right) \quad \text { and } \quad G(d)=G^{k}\left(u_{1}\right),
$$

for some $k \in \mathbb{N}$.

$$
F(b) \in\left(F^{k+1}\left(t_{1}\right), F^{k}\left(t_{1}\right)\right) \quad \text { and } \quad G(d) \in\left(G^{k+1}\left(u_{1}\right), G^{k}\left(u_{1}\right)\right)
$$


Assume that $F \in \mathcal{M}_{r,-}(a, b)$ and $G \in \mathcal{M}_{r,-}(c, d)$. Then $F$ and $G$ are conjugate if and only if either

$$
F(a)=b \quad \text { and } \quad G(c)=d,
$$

or

$$
F(a)=F^{k}\left(t_{r}\right) \quad \text { and } \quad G(c)=G^{k}\left(u_{r}\right),
$$

or

for some $k \in \mathbb{N}$.

$$
F(a) \in\left(F^{k}\left(t_{r}\right), F^{k+1}\left(t_{r}\right)\right) \quad \text { and } \quad G(c) \in\left(G^{k}\left(u_{r}\right), G^{k+1}\left(u_{r}\right)\right)
$$

The authors described also the construction of all homeomorphisms conjugating $F$ and $G$. Roughly speaking, all of them, that is homeomorphic solutions of equation (1.1), "depend on an arbitrary function". In other words, (almost) arbitrary continuous strictly monotonic function, defined on a suitable interval, can be extended on $[a, b]$ to the desired homeomorphic conjugacy.

The quite recent paper [44] by Yong-Guo Shi, Lin Li, and Zb. Leśniak brings a description of the conjugacy in larger classes of functions, not necessarily of height 1 . Given $r \in \mathbb{N}$ and $\kappa \in \mathbb{N} \cup\{\infty\}$ the authors consider the classes $\mathcal{M}_{r,+}^{\kappa}(a, b)$ and $\mathcal{M}_{r,-}^{\kappa}(a, b)$ defined as follows: a function $F \in \mathcal{M}_{r}(a, b)$ with $S(F)=\left\{t_{1}, \ldots, t_{r}\right\}$, where $a<t_{1}<\ldots<t_{r}<b$, belongs to $\mathcal{M}_{r,+}^{\kappa}(a, b)$ if

$$
F\left(\left[a, t_{1}\right]\right) \subset\left[a, t_{1}\right], F(x)<x \text { for } x \in\left(t_{1}, b\right), \text { and } H(F)=\kappa ;
$$

similarly, $F \in \mathcal{M}_{r,-}^{\kappa}(a, b)$ if

$$
F\left(\left[t_{r}, b\right]\right) \subset\left[t_{r}, b\right], F(x)>x \text { for } x \in\left(a, t_{r}\right), \text { and } H(F)=\kappa .
$$

To formulate the main result of [44] we need some additional notation.

Let $F \in \mathcal{M}_{r}(a, b)$ with $S(F)=\left\{t_{1}, \ldots, t_{r}\right\}$, where $a=t_{0}<t_{1}<\ldots<t_{r}<t_{r+1}=b$ and let $\kappa \in \mathbb{N} \cup\{\infty\}$. If $F \in \mathcal{M}_{r,+}^{\kappa}(a, b)$ then for each $x \in[a, b]$ we define the sequence $L_{F}^{+}(x)=\left(l_{k}^{+}(x)\right)_{k \in \mathbb{N}_{0}}$ by

$$
l_{k}^{+}(x)= \begin{cases}0, & \text { if } \quad F^{k}(x) \in\left[t_{0}, t_{1}\right] \\ i, & \text { if } \quad F^{k}(x) \in\left(t_{i}, t_{i+1}\right], i=1, \ldots, r .\end{cases}
$$

Similarly, if $F \in \mathcal{M}_{r,-}^{\kappa}(a, b)$ then for each $x \in[a, b]$ we put

$$
l_{k}^{-}(x)= \begin{cases}0, & \text { if } \quad F^{k}(x) \in\left[t_{r}, t_{r+1}\right] \\ i, & \text { if } \quad F^{k}(x) \in\left[t_{i-1}, t_{i}\right), i=1, \ldots, r\end{cases}
$$

and $L_{F}^{-}(x)=\left(l_{k}^{-}(x)\right)_{k \in \mathbb{N}_{0}}$.

The following result can be found in [44]. It allows us to confine ourselves to the case $r_{1}=r_{2}$ and $\kappa_{1}=\kappa_{2}$ while studying the conjugacy of functions $F \in \mathcal{M}_{r_{1},+}^{\kappa_{1}}(a, b)$ and $G \in \mathcal{M}_{r_{2},+}^{\kappa_{2}}(c, d)$, or $F \in \mathcal{M}_{r_{1},-}^{\kappa_{1}}(a, b)$ and $G \in \mathcal{M}_{r_{2},-}^{\kappa_{2}}(c, d)$.

Fact 1.6. If $F \in \mathcal{M}_{r_{1}}(a, b)$ and $G \in \mathcal{M}_{r_{2}}(c, d)$ are conjugate, then $r_{1}=r_{2}$ and $H(F)=H(G)$.

Below is the main result of [44].

Theorem 1.7. Let $F \in \mathcal{M}_{r}(a, b)$ and $G \in \mathcal{M}_{r}(c, d)$ with $S(F)=\left\{t_{1}, \ldots, t_{r}\right\}$ and $S(G)=\left\{u_{1}, \ldots, u_{r}\right\}$, where $a, b, c, d \in \mathbb{R}, a=t_{0}<t_{1}<\ldots<t_{r}<t_{r+1}=b$ and $c=u_{0}<u_{1}<\ldots<u_{r}<u_{r+1}=d$. Assume that $F \in \mathcal{M}_{r,+}^{\kappa}(a, b)$ and $G \in \mathcal{M}_{r,+}^{\kappa}(c, d)$ for some $\kappa \in \mathbb{N} \cup\{\infty\}$. Then $F$ and $G$ are conjugate if and only if

$$
L_{F}^{+}\left(t_{i}\right)=L_{G}^{+}\left(u_{i}\right), \quad i=0,1, \ldots, r+1,
$$


and there exists a continuous strictly increasing function $\phi_{0}:\left[a, t_{1}\right] \rightarrow\left[c, u_{1}\right]$ conjugating $\left.F\right|_{\left[a, t_{1}\right]}$ and $\left.G\right|_{\left[c, u_{1}\right]}$ such that

$$
\begin{aligned}
& \text { if } \quad \kappa<\infty \quad \text { then } \quad \phi_{0}\left(F^{\kappa}\left(t_{i}\right)\right)=G^{\kappa}\left(u_{i}\right), \quad i=0,1, \ldots, r+1 \\
& \text { if } \quad \kappa=\infty \quad \text { then } \quad \phi_{0}\left(F^{\kappa_{0}}\left(t_{i}\right)\right)=G^{\kappa_{0}}\left(u_{i}\right), \quad i=0,1, \ldots, r
\end{aligned}
$$

where $\kappa_{0}=H\left(\left.F\right|_{\left[a, t_{r}\right]}\right)$.

Assume that $F \in \mathcal{M}_{r,-}^{\kappa}(a, b)$ and $G \in \mathcal{M}_{r,-}^{\kappa}(c, d)$ for some $\kappa \in \mathbb{N} \cup\{\infty\}$. Then $F$ and $G$ are conjugate if and only if

$$
L_{F}^{-}\left(t_{i}\right)=L_{G}^{-}\left(u_{i}\right), \quad i=0,1, \ldots, r+1
$$

and there exists a continuous strictly increasing function $\phi_{0}:\left[t_{r}, b\right] \rightarrow\left[u_{r}, d\right]$ conjugating $\left.F\right|_{\left[t_{r}, b\right]}$ and $\left.G\right|_{\left[u_{r}, d\right]}$ such that (1.2) holds, where $\kappa_{0}=H\left(\left.F\right|_{\left[a, t_{r}\right]}\right)$.

In both cases $\phi_{0}$ can be uniquely extended on $[a, b]$ to a homeomorphism conjugating $F$ and $G$.

Conjugacy and iterative roots are closely related. This can be well seen from the following consequence of Theorem 1.7, proved in the paper [44]. Observe that the assumptions imposed on $F$ here force the equality $H(F)=1$.

Corollary 1.8. Let $F \in \mathcal{M}_{r}(a, b)$ with $S(F)=\left\{t_{1}, \ldots, t_{r}\right\}$, where $a=t_{0}<t_{1}<\ldots<t_{r}<t_{r+1}=b$.

Assume that

$$
\text { if } i \text { is even, then } F\left(t_{i}\right)=t_{0} \text { and if } i \text { is odd, then } F\left(t_{i}\right)=F\left(t_{1}\right)
$$

for every $i \in\{0, \ldots, r+1\}$. Then for every $n \in \mathbb{N}$ the set $\Phi_{n}$ of conjugacies $\phi$ satisfying the equation $\phi \circ F=F^{n} \circ \phi$ is non-void. Moreover, $\left\{\phi^{-1} \circ F \circ \phi: \phi \in \Phi_{n}\right\}$ is the set of all iterative roots of order $n$ of $F$ belonging to the class $\mathcal{M}_{r,+}^{1}(a, b)$.

Assume that

$$
\text { if } \left.i \text { is even, then } F\left(t_{i}\right)=F\left(t_{r}\right)\right) \text { and if } i \text { is odd, then } F\left(t_{i}\right)=t_{r+1}
$$

for every $i \in\{0, \ldots, r+1\}$. Then for every $n \in \mathbb{N}$ the set $\Phi_{n}$ of conjugacies $\phi$ satisfying the equation $\phi \circ F=F^{n} \circ \phi$ is non-void. Moreover, $\left\{\phi^{-1} \circ F \circ \phi: \phi \in \Phi_{n}\right\}$ is the set of all iterative roots of order $n$ of $F$ belonging to the class $\mathcal{M}_{r,-}^{1}(a, b)$.

The equation $\phi \circ F=F^{n} \circ \phi$, used in the definition of the set $\Phi_{n}$ above, is of interest on its own. This is a particular case of the conjugacy equation

$$
\phi \circ F^{m}=F^{n} \circ \phi
$$

studied by J. Haneczok in [10] in the setting of formal power series; here $m, n \in \mathbb{N}$, or even $m, n \in \mathbb{Z}$ if $F$ is bijective.

\section{ITERATIVE ROOTS OF PIECEWISE MONOTONIC FUNCTIONS}

At the very begining remark the simple but important observation below, following from Fact 1.1.

Fact 2.1. Every continuous iterative root of a piecewise monotonic [strictly monotonic] function is also piecewise monotonic [strictly monotonic].

The paper [5] by A. Blokh, E. Coven, M. Misiurewicz and Zb. Nitecki is classical in the theory of iterative roots of piecewise monotonic functions. Here, however, we deal mainly with papers studying piecewise monotonic functions with respect to their nonmonotonicity heights.

If $H(F)=0$ then $F$ is strictly monotonic. In 1961, in the paper [17], Marek Kuczma gave a complete description of iterative roots in such a case (see also [18], [58] and [19]). Roughly speaking he found that: 
if $F$ is strictly increasing, then

- it has continuous strictly increasing roots of an arbitrary order,

- it has no decreasing roots of any odd order,

- necessary and sufficient conditions to have continuous strictly decreasing roots of a given even order are known;

if $F$ is strictly decreasing, then

- it has no increasing roots of any order,

- it has continuous strictly decreasing roots of an arbitrary odd order,

- it has no decreasing roots of any even order.

If $F$ has a root of order at least 2, then $F$ has infinitely many roots of the same order: all of them depend on an arbitrary function which means that each can be constructed by an extension of an (almost) arbitrary continuous strictly monotonic function defined on a suitable subinterval of $[a, b]$. Notice also that strictly increasing roots of continuous strictly increasing functions were determined yet by U.T. Bödewadt [8] in 1944 .

The case $H(F)=1$ is also well studied (cf. [72] by Weinian Zhang and [25] by Lin Li, Dilian Yang and Weinian Zhang) and can be reduced to the monotonic case in the following way. By Fact 1.3 we know that the function $F$ is not monotonic on the interval $[a, b]$ but it is monotonic on the interval $F([a, b])$. The maximal interval $K(F)$, containing $F([a, b])$ and such that $F$ is monotonic on it, is called the characteristic interval of $F$. Since $F(K(F)) \subset K(F)$ and $H\left(\left.F\right|_{K(F)}\right)=0$ we can use Kuczma's theorem and find a continuous root $f$ of $\left.F\right|_{K(F)}$. This can be extended on $[a, b]$ to a continuous root of $F$. The method of determining iterative roots of piecewise monotonic functions by using their characteristic intervals was proposed and described in Chinese by Jingzhong Zhang and Lu Yang [68] in 1983, and then improved by Weinian Zhang [72] in 1997. Investigation of this method in the case $H(F)=1$ has been recently completed by Liu Liu and Weinian Zhang in the paper [27]. The authors proved there that actually every continuous iterative root of $F$ can be obtained by extension from the characteristic interval.

Now we are interested in the more complicated case $H(F) \geq 2$. Then, as Weinian Zhang proved in [72], the function $F$ has no continuous iterative roots of order $r$ greater than $\# S(F)$. So the problem is:

Does $F \in \mathcal{P} \mathcal{M}(a, b)$ with $H(F) \geq 2$ have an iterative root of order $r$ for $r \leq \# S(F)$ ?

It seems that in the case $\# S(F)=2$ this problem was solved by Taixiang Sun and Hongjian Xi [48] and Taixiang Sun [47]. However, unfortunately it is not so easy to check it, since their results have been formulated in a sophisticated and not immediate form. Moreover, both papers are not available for a wide audience, as they are written in Chinese. The case $\# S(F) \geq 3$ was discussed for a special class of piecewise monotonic functions by Guangyuan Zhang [67], also only in Chinese.

The results presented below originate in the paper [26]. Consider the case $r=\# S(F)$, i.e. the order of a desired root equals the number of turning points of $F$. Assume that $r \geq 2$ and write

$$
S(F)=\left\{t_{1}, \ldots, t_{r}\right\}
$$

where $a<t_{1}<\ldots<t_{r}<b$.

The following lemma shows that if $F \in \mathcal{P} \mathcal{M}(a, b)$ has a root $f \in \mathcal{P} \mathcal{M}(a, b)$ of order $r$, then $f$ has a very special form.

Lemma 2.2. Let $F \in \mathcal{P} \mathcal{M}(a, b)$ with $H(F) \geq 2$. If $f \in \mathcal{P} \mathcal{M}(a, b)$ is an iterative root of order $r=\# S(F)$ of $F$, then $f$ has exactly one turning point. Moreover, one of the following possibilities holds:

$\mathcal{T}_{1}$. either $S(f)=\left\{t_{1}\right\}$, the function $f$ reaches the minimum value at $t_{1}$ and $f\left(t_{1}\right)<t_{1}$, 


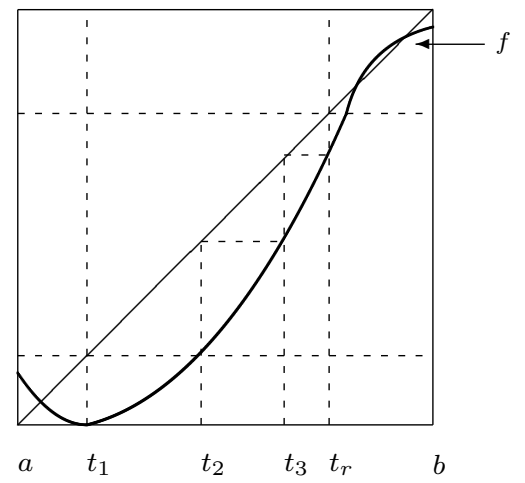

case $\mathcal{T}_{1} . S(f)=\left\{t_{1}\right\}$

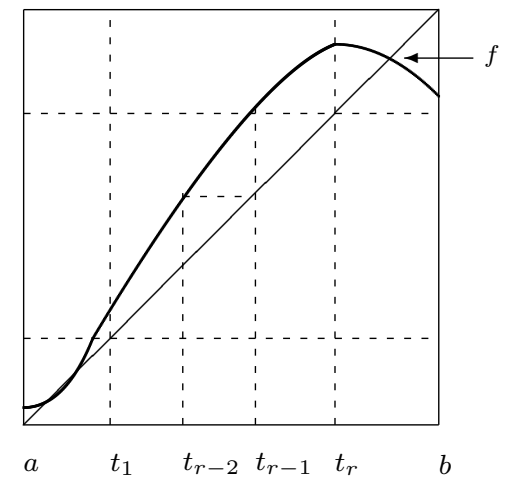

case $\mathcal{T}_{1} . S(f)=\left\{t_{r}\right\}$

or $S(f)=\left\{t_{r}\right\}$, the function $f$ reaches the maximum value at $t_{r}$ and $f\left(t_{r}\right)<t_{r}$.

$\mathcal{T}_{2}$. either $S(f)=\left\{t_{1}\right\}$, the function $f$ reaches the maximum value at $t_{1}$ and $f\left(t_{1}\right)>t_{1}$,

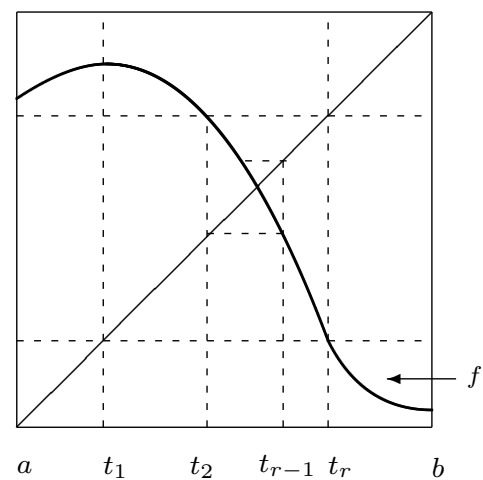

case $\mathcal{T}_{2} . S(f)=\left\{t_{1}\right\}$

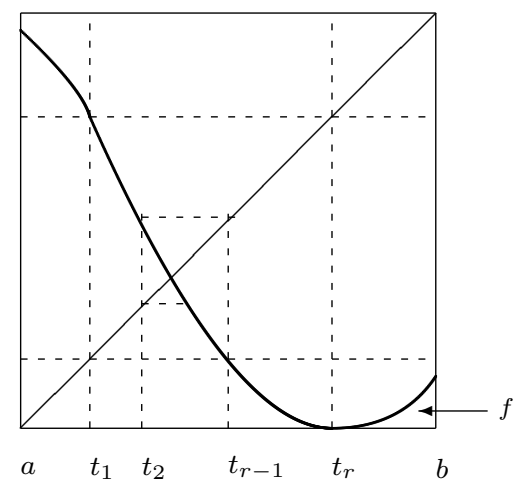

case $\mathcal{T}_{2} . S(f)=\left\{t_{r}\right\}$

or $S(f)=\left\{t_{r}\right\}$, the function $f$ reaches the minimum value at $t_{r}$ and $f\left(t_{r}\right)<t_{r}$.

We know a full characterization of those functions $F \in \mathcal{P} \mathcal{M}(a, b)$ that have a root of order $r=\# S(F)$ of type $\mathcal{T}_{1}$. To formulate the main result of this section we need one notion more. This is a modification of the notions of conjugacy of intervals, and semiregularity and regularity of points, introduced by M. Kuczma in [17, Def. II-IV] (see also [18, Def. on p. 301]).

Let $I$ be a compact interval. A strictly increasing function $\phi$ mapping $I$ into itself is said to be a reversing correspondence if there are a $\xi \in \operatorname{Fix} \phi$, i.e. a fixed point of $\phi$, and a strictly decreasing function $\omega$ mapping Fix $\phi$ onto itself such that $\omega(\xi)=\xi$ and the expression $\phi(x)-x$ has opposite signs in the intervals $\left(\xi_{1}, \xi_{2}\right)$ and $\left(\omega\left(\xi_{2}\right), \omega\left(\xi_{1}\right)\right)$ for every $\xi_{1}, \xi_{2} \in \operatorname{Fix} \phi$ with $\xi_{1}<\xi_{2}$ and $\left(\xi_{1}, \xi_{2}\right) \cap \operatorname{Fix} \phi=\varnothing$.

The result mentioned above reads as follows.

Theorem 2.3. Let $F \in \mathcal{P} \mathcal{M}(a, b)$ with $H(F) \geq 2$ and assume that $\# S(F) \geq 2$. Then $F$ has an iterative root of order $r=\# S(F)$ of type $\mathcal{T}_{1}$ if and only if one of the following conditions is fulfilled: 
(i) $r$ is even, $\left.F\right|_{\left[a, t_{1}\right]}$ is a reversing correspondence and either

$$
F(a)=F\left(t_{2}\right)=\ldots=F\left(t_{r-2}\right)=F\left(t_{r}\right)=a \text { and } F\left(t_{1}\right)=F\left(t_{3}\right)=\ldots=F\left(t_{r-1}\right)=t_{1} ; \quad \text { (even-eq) }
$$

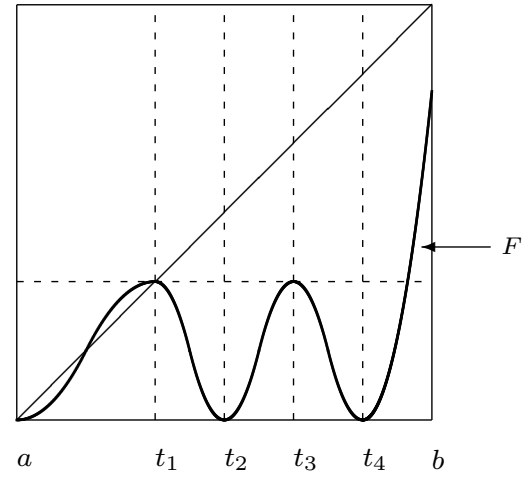

case (even-eq)

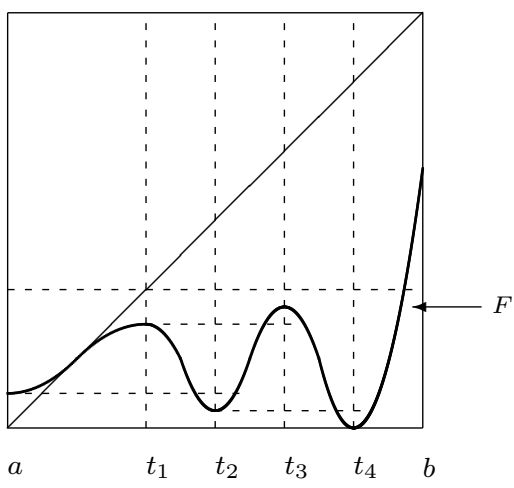

case (even-ineq)

or

$$
F(a)>F\left(t_{2}\right)>\ldots>F\left(t_{r-2}\right)>F\left(t_{r}\right) \text { and } F\left(t_{1}\right)<F\left(t_{3}\right)<\ldots<F\left(t_{r-1}\right)<t_{1}, \quad \text { (even-ineq) }
$$

(ii) $r$ is odd, $\left.F\right|_{\left[a, t_{1}\right]}$ is decreasing and either

$$
F(a)=F\left(t_{2}\right)=\ldots=F\left(t_{r-1}\right)=t_{1} \text { and } F\left(t_{1}\right)=F\left(t_{3}\right)=\ldots=F\left(t_{r}\right)=a
$$

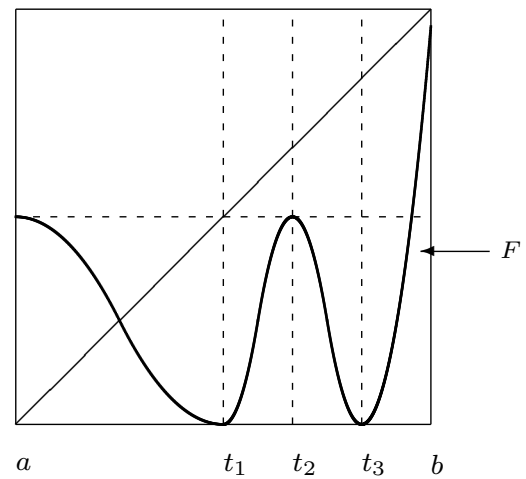

case (odd-eq)

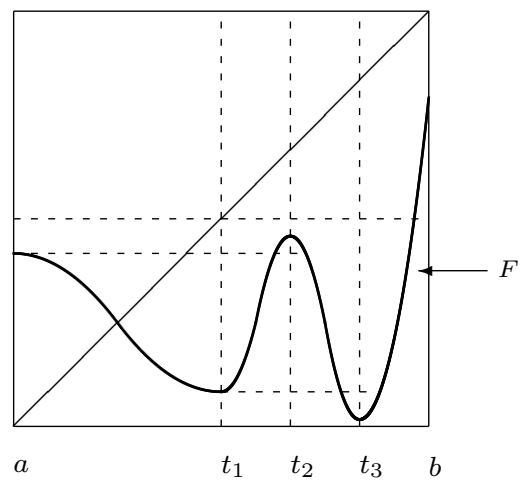

case (odd-ineq)

or

$$
F(a)<F\left(t_{2}\right)<\ldots<F\left(t_{r-1}\right)<t_{1} \text { and } F\left(t_{1}\right)>F\left(t_{3}\right)>\ldots>F\left(t_{r}\right),
$$

(odd-ineq)

(iii) $r$ is even, $\left.F\right|_{\left[t_{r}, b\right]}$ is a reversing correspondence and either

$$
F(b)<F\left(t_{r-1}\right)<\ldots<F\left(t_{3}\right)<F\left(t_{1}\right) \text { and } F\left(t_{r}\right)>F\left(t_{r-2}\right)>\ldots>F\left(t_{2}\right)>t_{r}
$$


or

$$
F(b)=F\left(t_{r-1}\right)=\ldots=F\left(t_{3}\right)=F\left(t_{1}\right)=b \text { and } F\left(t_{r}\right)=F\left(t_{r-2}\right)=\ldots=F\left(t_{2}\right)=t_{r}
$$

(iv) $r$ is odd, $\left.F\right|_{\left[t_{r}, b\right]}$ is decreasing and either

$$
F(b)>F\left(t_{r-1}\right)>\ldots>F\left(t_{2}\right)>t_{r} \text { and } F\left(t_{r}\right)<F\left(t_{r-2}\right)<\ldots<F\left(t_{1}\right),
$$

or

$$
F(b)=F\left(t_{r-1}\right)=\ldots=F\left(t_{2}\right)=t_{r} \text { and } F\left(t_{r}\right)=F\left(t_{r-2}\right)=\ldots=F\left(t_{1}\right)=b
$$

We complete this section with posing two problems:

1. Characterize all the functions $F \in \mathcal{P} \mathcal{M}(a, b)$ having a root of order $r=\# S(F)$ of type $\mathcal{T}_{2}$.

2. Answer the question: is it possible to apply Theorem 1.7 to prove Theorem 2.3 and/or to solve the problem 1.

\section{Stability problems for iterative Roots. Approximate roots}

Iterative roots have various applications, among other in theory of neutral networks and in graph theory. For that reason we need algorithms for their numerical computation. In general, it is difficult to find them. One of possibilities is to give them for polygonal functions at first, and then consider their approximation to continuous functions in the general case. The errors from the numerical computation and approximation highly affect the validity of the computation, which requires the stability of iterative roots. An algorithm for computing square roots of increasing polygonal mappings was given by J. Kobza in [16]. Then it was generalized by Wanxiong Zhang and Weinian Zhang [70] to not only increasing polygons, but also decreasing polygons and some non-monotonic ones, and to roots of an arbitrary order.

We start with a special kind of stability, which is Hyers-Ulam stability. Roughly speaking, a functional equation is stable in the sense of Hyers and Ulam if any function approximately satisfying the equation is an approximate solution, i.e. is close to a solution. This concept of stability has often be employed successfully to approximate a real solution by a sequence of approximate solutions. In 2007 Bing Xu and Weinian Zhang [61] established the Hyers-Ulam stability of increasing roots of continuous strictly increasing functions. Actually this is a local result as the domain of roots, being an open neighbourhood of the fixed point, cannot be extended to include another fixed point.

Theorem 3.1. Let $n \geq 2$ be an integer, $F:[a, b] \rightarrow[a, b]$ be a continuous strictly increasing function and let $g:[a, b] \rightarrow[a, b]$ satisfy the Lipschitz condition:

$$
\left|g\left(x_{1}\right)-g\left(x_{2}\right)\right| \leq c\left|x_{1}-x_{2}\right|, \quad x_{1}, x_{2} \in[a, b]
$$

with a $c \in(0, \infty)$ such that $\vartheta:=\sum_{i=1}^{n-1} c^{i}<1$.

If $a<F(x)<x$ for each $x \in(a, b]$, there is an $x_{0} \in[a, b]$ such that

$$
a<F\left(x_{0}\right)=g^{n}\left(x_{0}\right)<g^{n-1}\left(x_{0}\right)<\ldots<g\left(x_{0}\right)<x_{0},
$$

the function $g$ strictly increases in $\left[g^{n-1}\left(x_{0}\right), x_{0}\right]$, and

$$
\left|g^{n}(x)-F(x)\right| \leq \delta, \quad x \in\left[a, x_{0}\right]
$$


for a $\delta \in(0, \infty)$, then there exists a continuous strictly increasing function $f:\left[a, x_{0}\right] \rightarrow\left[a, x_{0}\right]$ such that $a<f(x)<x$ for each $x \in\left[a, x_{0}\right]$,

$$
|f(x)-g(x)| \leq \frac{\delta}{1-\vartheta}, \quad x \in\left[a, x_{0}\right],
$$

end equality (0.1) holds for every $x \in\left[a, x_{0}\right]$.

If $x<F(x)<b$ for each $x \in[a, b)$, there is an $x_{0} \in[a, b]$ such that

$$
x_{0}<g\left(x_{0}\right)<\ldots<g^{n-1}\left(x_{0}\right)<g^{n}\left(x_{0}\right)=F\left(x_{0}\right)<b,
$$

the function $g$ strictly increases in $\left[x_{0}, g^{n-1}\left(x_{0}\right)\right]$, and

$$
\left|g^{n}(x)-F(x)\right| \leq \delta, \quad x \in\left[x_{0}, b\right],
$$

for a $\delta \in(0, \infty)$, then there exists a continuous strictly increasing function $f:\left[x_{0}, b\right] \rightarrow\left[x_{0}, b\right]$ such that $x<f(x)<b$ for each $x \in\left[x_{0}, b\right]$,

$$
|f(x)-g(x)| \leq \frac{\delta}{1-\vartheta}, \quad x \in\left[x_{0}, b\right],
$$

end equality (0.1) holds for every $x \in\left[x_{0}, b\right]$.

The Hyers-Ulam stability of continuous roots, provided by Theorem 3.1, cannot fully support the algorithm given in [70] for the following two reasons:

- it is hard to construct a polygonal solution, following the algorithm presented in [70], satisfying $\vartheta<1$, where $\vartheta=\sum_{i=1}^{n-1} c^{i}$ as in Theorem 3.1 ;

- the assumption $\vartheta<1$ obstructs obtaining iterative roots with more than one fixed point.

These disadvantages do not occur while considering the global stability of continuous roots, proposed in the paper [74] by Wenmeng Zhang and Weinian Zhang. To see this let us recall a classical result dealing with determining increasing roots of strictly increasing self-mappings of $[a, b]$ (see [19, Th. 11.2.2], also [18]).

Denote by $\mathcal{H}_{+}(a, b)$ and $\mathcal{H}_{-}(a, b)$ the sets of strictly increasing functions $F$ mapping $[a, b]$ onto itself and satisfying the conditions.

$$
a<F(x)<x, x \in(a, b), \quad \text { and } \quad x<F(x)<b, x \in(a, b),
$$

respectively.

Let $F \in \mathcal{H}_{+}(a, b)$ and take arbitrarily given $n$ initial points $x_{0}, \ldots, x_{n} \in(a, b)$ such that

$$
a<F\left(x_{0}\right)<x_{n-1}<\ldots<x_{0}<b .
$$

Then (see [19, Th. 11.2.2], also [18]) each continuous strictly increasing function $f_{0}$, mapping $\left[x_{n-1}, x_{0}\right]$ onto $\left[F\left(x_{0}\right), x_{1}\right]$ and satisfying

$$
f_{0}\left(x_{i-1}\right)=x_{i}, \quad i \in\{1, \ldots, n-1\},
$$

can be uniquely extended on $[a, b]$ to an iterative root $f \in \mathcal{H}_{+}(a, b)$ of $F$ of order $n$. Similarly, if $F \in \mathcal{H}_{-}(a, b)$ and

$$
a<x_{0}<\ldots<x_{n-1}<F\left(x_{0}\right)<b,
$$


then every continuous strictly increasing $f_{0}$, mapping $\left[x_{0}, x_{n-1}\right]$ onto $\left[x_{1}, F\left(x_{0}\right)\right]$ and satisfying (3.2), can be uniquely extended on $[a, b]$ to a root $f \in \mathcal{H}_{-}(a, b)$ of $F$ of order $n$. Any such $f_{0}$ will be called an initial function associated to $F$ and $x_{0}, \ldots, x_{n-1}$, no matter $(3.1)$ or $(3.3)$ holds.

In [74] the authors gave approximations of iterative roots of monotonic functions, positively answering the following question:

Given a sequence $\left(F_{k}\right)_{k \in \mathbb{N}}$ tending, in a sense, to $F$, can we find a root $f_{k}$ of $F_{k}$ of order $n$ for each $k \in \mathbb{N}$ such that $\left(f_{k}\right)_{k \in \mathbb{N}}$ tends to the root of $F$ of order $n$, associated with a given initial function?

Actually the authors of [74] proved three theorems dealing with, as in classical Kuczma's theory, increasing roots of strictly increasing functions, decreasing roots of strictly increasing functions, and decreasing roots of strictly decreasing functions. Below is a result concerning the first case only.

Given any $r \in \mathbb{N}$ denote by $\mathcal{C}^{r}(a, b)$ the space of all $\mathcal{C}^{r}$ self-mappings of the interval $[a, b]$, endowed with the norm \|\|$_{r}$ given by

$$
\|h\|_{r}=\sum_{i=0}^{r} \sup \left\{\left|h^{(i)}(x)\right|: x \in[a, b]\right\} .
$$

Theorem 3.2. Let $F \in \mathcal{H}_{+}(a, b)$ and $x_{0}, \ldots, x_{n-1} \in(a, b)$ satisfy inequalities (3.1), and let $f_{0}:\left[x_{n-1}, x_{0}\right] \rightarrow$ $\left[F\left(x_{0}\right), x_{1}\right]$ be a function associated to $F$ and $x_{0}, \ldots, x_{n-1}$. Let $\left(F_{k}\right)_{k \in \mathbb{N}}$ be a sequence of functions from $\mathcal{H}_{+}(a, b)$ such that $\lim _{k \rightarrow \infty}\left\|F_{k}-F\right\|_{0}=0$. Then, for every $k \in \mathbb{N}$ large enough, there exists an initial function $f_{k, 0}:\left[x_{n-1}, x_{0}\right] \rightarrow\left[F_{k}\left(x_{0}\right), x_{1}\right]$, associated to $F_{k}$ and $x_{0}, \ldots, x_{n-1}$, which can be extended on $[a, b]$ to a root $f_{k} \in \mathcal{H}_{+}(a, b)$ of $F_{k}$ of order $n$; moreover,

$$
\lim _{k \rightarrow \infty}\left\|f_{k, 0}-f_{0}\right\|_{0}=0 \quad \text { and } \quad \lim _{k \rightarrow \infty}\left\|f_{k}-f\right\|_{0}=0
$$

where $f \in \mathcal{H}_{+}(a, b)$ is the unique extension of $f_{0}$ to a root of $F$ of order $n$.

Let $F \in \mathcal{H}_{-}(a, b)$ and $x_{0}, \ldots, x_{n-1} \in(a, b)$ satisfy inequalities $(3.3)$, and let $f_{0}:\left[x_{0}, x_{n-1}\right] \rightarrow\left[x_{1}, F\left(x_{0}\right)\right]$ be a function associated to $F$ and $x_{0}, \ldots, x_{n-1}$. Let $\left(F_{k}\right)_{k \in \mathbb{N}}$ be a sequence of functions from $\mathcal{H}_{-}(a, b)$ such that $\lim _{k \rightarrow \infty}\left\|F_{k}-F\right\|_{0}=0$. Then, for every $k \in \mathbb{N}$ large enough, there exists an initial function $f_{k, 0}:\left[x_{0}, x_{n-1}\right] \rightarrow$ $\left[x_{1}, F_{k}\left(x_{0}\right)\right]$, associated to $F_{k}$ and $x_{0}, \ldots, x_{n-1}$, which can be extended on $[a, b]$ to a root $f_{k} \in \mathcal{H}_{-}(a, b)$ of $F_{k}$ of order $n$; moreover, (3.4) holds, where $f \in \mathcal{H}_{-}(a, b)$ is the unique extension of $f_{0}$ to a root of $F$ of order $n$.

Now we pass to stability problems in the class $\mathcal{C}^{1}$. Unexpectedly the situation changes now. We present here two quite recent results in that direction proved in the paper [73]. We start with a particular case of a classical result proved by Marek Kuczma and Andrzej Smajdor in the paper [20].

For every $\lambda \in(0,1)$ define $\mathcal{H}_{\lambda}^{2}(a, b)$ as the class of functions $h \in \mathcal{C}^{2}(a, b)$ such that either

$$
h(x)<x \quad \text { and } \quad h^{\prime}(x)>0 \quad \text { for each } \quad x \in(a, b], \quad \text { and } \quad h^{\prime}(a)=\lambda,
$$

or

$$
h(x)>x \quad \text { and } \quad h^{\prime}(x)>0 \quad \text { for each } \quad x \in[a, b), \quad \text { and } \quad h^{\prime}(b)=\lambda .
$$

The following can be easily deduced from the mentioned result of Kuczma and Smajdor.

Let $\lambda \in(0,1)$ and $F \in \mathcal{H}_{\lambda}^{2}(a, b)$. Then, for every $n \in \mathbb{N}$, the function $F$ has a unique iterative root $f \in \mathcal{C}^{1}(a, b)$ of order $n$. Moreover,

$$
f(x)=\varphi^{-1}\left(\lambda^{1 / n} \varphi(x)\right), \quad x \in[a, b],
$$

where $\varphi:[a, b] \rightarrow \mathbb{R}$ is the principle solution of Schröder's equation

$$
\varphi(F(x))=\lambda \varphi(x)
$$


given by

$$
\varphi(x)=\lim _{k \rightarrow \infty} \frac{F^{k}(x)}{\lambda^{k}} .
$$

The following result gives a local stability of roots of class $\mathcal{C}^{1}$.

Theorem 3.3. Let $\lambda \in(0,1), F \in \mathcal{H}_{\lambda}^{2}(a, b)$, and let $\left(F_{k}\right)_{k \in \mathbb{N}}$ be a sequence of functions from $\mathcal{H}_{\lambda}^{2}(a, b)$. If

$$
\lim _{k \rightarrow \infty}\left\|F_{k}-F\right\|_{2}=0
$$

then

$$
\lim _{k \rightarrow \infty}\left\|f_{k}-f\right\|_{1}=0
$$

where $f_{k} \in \mathcal{C}^{1}(a, b)$ and $f \in \mathcal{C}^{1}(a, b)$ are the unique iterative roots of a given order of $F_{k}$ and $F$, respectively.

Rather unexpectedly the situation changes dramatically if the domain contains two (or more) fixed points of the given function. Then we come to a global $\mathcal{C}^{1}$ unstability, described by the following theorem. Notice that the functions $F$ and $F_{k}, k \in \mathbb{N}$, may be of class $\mathcal{C}^{r}$ there, where $r \in \mathbb{N}$ is arbitrary. Nevertheless, $F_{k}$ 's have no roots of order greater than 1 in the class $\mathcal{C}^{1}$, which for $r \geq 2$ is larger than $\mathcal{C}^{r}$.

Theorem 3.4. Let $r \in \mathbb{N}$ and let $F \in \mathcal{C}^{r}(a, b)$ satisfy the conditions $F(a)=a, F(b)=b, F^{\prime}(a), F^{\prime}(b) \in \mathbb{R} \backslash\{0,1\}$, $F(x) \neq x$ and $F^{\prime}(x)>0$ for every $x \in(a, b)$. Then there is a sequence $\left(F_{k}\right)_{k \in \mathbb{N}}$ of functions from $\mathcal{C}^{r}(a, b)$ such that

$$
\lim _{k \rightarrow \infty}\left\|F_{k}-F\right\|_{r}=0
$$

and having no $\mathcal{C}^{1}$ iterative root of any order greater than 1.

Several results, quite similar to Theorem 3.4 but in a completely different formal power series setting, were proved by L. Reich (see, for instance, [37] and [38]). There the local distribution of iterable and non-iterable formal power series transformations with respect to the order topology and the weak topology is investigated.

\section{Multifunction CASE}

It is symptomatic that the existence of iterative roots is rather an exceptional property. And this concerns both the purely set-theoretic case (cf., for instance, [75] by G. Zimmermann, but also [41] by R. E. Rice, B. Schweizer and A. Sklar) and the case of regular mappings, no matter we have in mind the continuity (see [11] by P.D. Humke and M. Laczkovich, [45] by K. Simon, and [4] by A.M. Blokh), or the holomorphicity (cf. [6] by $\mathrm{S}$. Bogatyi); nonexistence of roots is also typical for some regular functions which was estabilished by Weinian Zhang [71]. To avoid this inconvenience there have been many ideas how to extend the notion of iterative root. One of those initial trials, connected with the notion of so-called phantom root, was made by Gy. Targonski (cf., among others, [59] and [58]); his concept of phantom root and phantom iterate was taken up by J. Schwaiger, soon after the original proposal; a very similar idea goes back to S. Kurepa. Another, a newer one, involving setvalued functions as roots of single valued functions, was proposed by T. Powierża [33]- [35], also [13]. Namely, given a set $X$ and an integer $n \geq 2$ a multifunction $f: X \rightarrow 2^{X}$ is called a set-valued iterative root of $F: X \rightarrow 2^{X}$ of order $n$ if

$$
F(x) \in f^{n}(x), \quad x \in X,
$$

where $f^{0}(x):=\{x\}$ and $f^{k+1}(x)=\bigcup_{y \in f^{k}(x)} f(y)$ for each $k \in \mathbb{N}_{0}$ (cf. [35]). Of course the point is to determine the smallest (if it exists), in a sense, such a root.

Maybe the best approach to the problem of generalized iterative roots would be to replace single-valued functions by set-valued functions in equation (0.1), both for $F$ and $f$. However, such a formulation of the problem leads to many difficulties. Some complications can be already seen in the following simplest multifunction case. 
As the class of continuous self-mappings having an iterative root is small, one can enlarge it a little bit by admitting functions with some discontinuities. At the very beginning one can consider strictly monotonic mappings with only one point of discontinuity. A function with a unique jump can be identified with a multifunction with a unique set-valued point, where the jump of the function is replaced by the set-value of the multifunction. This problem was investigated only for square roots in the papers [14] and [24]. To formulate a typical result proved there let me introduce some notation and recall a terminology.

Fix a point $c \in[a, b]$ and denote by $\mathcal{F}_{c}(a, b)$ the set of all multifunctions $F:[a, b] \rightarrow 2^{[a, b]}$ such that \#F(c)>1 and $F(x)$ is a singleton whenever $x \in[a, b] \backslash\{c\}$. A multifunction $F:[a, b] \rightarrow 2^{[a, b]}$ is said to be upper semicontinuous at a point $x_{0} \in[a, b]$ if for every open set $V \subset[a, b]$ with $F\left(x_{0}\right) \subset V$ there exists a neighbourhood $U \subset[a, b]$ of $x_{0}$ such that $F(U) \subset V$; it is called upper semicontinuous if it is upper semicontinuous at every point of $[a, b]$. We also say that $F$ is strictly increasing if

$$
\sup F\left(x_{1}\right)<\inf F\left(x_{2}\right), \quad x_{1}, x_{2} \in[a, b], \quad x_{1}<x_{2},
$$

and strictly decreasing if

$$
\inf F\left(x_{1}\right)>\sup F\left(x_{2}\right), \quad x_{1}, x_{2} \in[a, b], \quad x_{1}<x_{2} .
$$

Multifunctions which are either strictly increasing, or strictly decreasing are called strictly monotonic.

The following result gives a necessary and sufficient condition for the existence of strictly increasing and upper semicontinuous multifunctions from the class $\mathcal{F}_{c}(a, b)$; we confine ourselves here only to the simpler case of strictly increasing roots. The case of strictly decreasing roots is also treated in the paper [24] but the description is more complicated.

Given a real number $c$ for every set $A \subset \mathbb{R}$ put $A_{-}=A \cap(-\infty, c)$ and $A_{+}=A \cap(c, \infty)$.

Theorem 4.1. Let $F \in \mathcal{F}_{c}(a, b)$ be strictly increasing and upper semicontinuous. Then $F$ has a strictly increasing and upper semicontinuous square iterative root if and only if either $c \notin F([a, b])$, or $c \in F(c)$ and there exist strictly increasing continuous functions $\gamma_{-}:[a, c) \rightarrow[a, c)$ and $\gamma_{+}:(c, b] \rightarrow(c, b]$ satisfying equations $\gamma_{-}^{2}=\left.F\right|_{[a, c)}, \gamma_{+}^{2}=\left.F\right|_{(c, b]}$, and a set $M \subset[a, b]$ such that $c \in M, F(c)_{-}=\gamma_{-}\left(M_{-}\right) \cup M_{-}$and $F(c)_{+}=\gamma_{+}\left(M_{+}\right) \cup M_{+}$.

The paper [24] provides also a full construction of all the roots; roughly speaking, as previously, roots "depend on an arbitrary function" whenever they exist.

The idea to replace single-valued functions by multifunctions has been examined also in the embedding problem in iteration theory, strictly related to the problem of iterative roots. Among results in this direction, published during the last decade, there are those by G. Eydzińska [28]- [31] as well as [63] due to M.C. Zdun (see also the very recent survey article [65] by him, published at the same volume).

Up to now we have no notion of a generalized iterative root leading to really satisfactory results. However, no doubt that such notions are needed to build a theory in which more functions have such weak or generalized roots. It seems that at least three problems should be solved in the first instance:

1. Find conditions sufficient for the existence of the smallest set-valued root in the sense of Powierża and describe its construction.

2. Develope the idea of finding set-valued roots of multifunctions, specifying suitable definitions of basic notions and studying their fundamental properties.

3. Collect examples coming from life and applications, confirming the necessity of considering set-valued analogues while looking for iterative roots. 


\section{Not Discussed Although important}

Because of the personal choice of papers and results presented here, and the limited volume of this survey article, a number of topics has not been even touched. To right a wrong a little bit we at least mention three of them.

(i) Iterative roots of formal power series. A complete answer to the question, if a formal power series $F$ of the form

$$
F(x)=\rho x+c_{2} x^{2}+\ldots
$$

has an iterative root of a fixed order $n$, was given in the paper [42] by J. Schwaiger. If $f$ of the form $f(x)=$ $\sigma x+d_{2} x^{2}+\ldots$ is such a root, then, clearly,

$$
\sigma^{n}=\rho .
$$

L. Reich [36] fully answered the question whether the series (5.1) (given in its semicanonical form) has a root of order $n$ with a given multiplier $\sigma$ being a fixed solution of (5.2). Both the results are the main points of the paper [7] by S. Bogatyi who, among others, reformulated Reich's theorem in invariant form and gave its another proof, using ideas essentially different from those presented in [36].

(ii) Iterative roots of self-mappings of the circle. It seems that it was M.C. Zdun who initiated an intensive study these topics in the paper [62] in 2000. There is an earlier one, viz. [32] by Jie Hua Mai, but it is hardly obtainable as written in Chinese; moreover, it sketches the proofs only. Among papers devoted to this subject there are [12] and, first of all, the cycle of papers [49]- [56] by P. Solarz. The main problem is here to find criteria for the existence (and sometimes the uniqueness) of iterative roots of homeomorphisms of the circle.

(iii) Higher dimensions. Iteration problems for power series transformations in higher dimensions were intensively investigated by L. Reich and J. Schwaiger (cf., for instance, [39] and [40]) who followed the work of Shl. Sternberg [57].

What concerns recent results, mainly the papers [21]- [23] by Zb. Leśniak should be pointed out; they concern iterative roots of self-mappings of subsets of the real plane, among others Brouwer homeomorphisms, and perfectly show serious difficulties in the research in such a case.

The problem of iterative roots of functions mapping a subset of $\mathbb{R}^{p}$ into itself is only touched up to now. The reader is referred to the recent paper [64] by M.C. Zdun dealing, among others, with this problem.

Acknowledgement. The author is indebted to the referee for valuable remarks and comments to this survey.

\section{REFERENCES}

[1] Ch. Babbage, Essay towards the calculus of functions, Philosoph. Transact. 1815, 389-423; Essay towards the calculus of functions. II, ibid. 1816, 179-256.

[2] K. Baron, Recent results in a theory on functional equations in a single variable, Series Mathematicae Catoviciensis et Debreceniensis (Seminar LV) 15 (2003), 16pp., 31.01.2003, http:/www.math.us.edu.pl/smdk/papers2.html

[3] K. Baron and W. Jarczyk, Recent results on functional equations in a single variable, perspectives and open problems, Aequationes Math. 61 (2001), 1-48.

[4] A.M. Blokh, The set of all iterates is nowhere dense in $[(0,1],[0,1])$, Trans. Amer. Math. Soc. 333 (1992), 787-798.

[5] A. Blokh, E. Coven, M. Misiurewicz and Zb. Nitecki, Roots of continuous piecewise monotone maps of an interval, Acta Math. Univ. Comenian. (NS) 60 (1991), 3-10.

[6] S. Bogatyi, On the nonexistence of iterative roots, Topology Appl. 76 (1997), 97-123.

[7] S. Bogatyi, A topological view on a problem on formal series, Aequationes Math. 56 (1998), 18-26.

[8] U.T. Bödewadt, Zur Iteration reeller Funktionen, Math. Z. 49 (1944), 497-516.

[9] K. Ciepliński and M.C. Zdun, On uniqueness of conjugacy of continuous and piecewise monotonic functions, Fixed Point Theory Appl. 2009, Art. ID 230414, 11pp.

[10] J. Haneczok, Conjugacy type problems in the ring of formal power series, Grazer Math. Ber. 353, Graz, 2009. 
[11] P.D. Humke and M. Laczkovich, The Borel structure of iterates of continuous functions, Proc. Edinburgh Math. Soc. 32 (1986), 483-494.

[12] W. Jarczyk, Babbage equation on the circle, Publ. Math. Debrecen 63 (2003), 389-400.

[13] W. Jarczyk and T. Powierża, On the smallest set-valued iterative roots of bijections, Internat. J. Bifur.Chaos Appl. Sci. Engrs. 13 (2003), 1889-1893.

[14] W. Jarczyk and Weinian Zhang, Also set-valued functions do not like iterative roots, Elem. Math. 62 (2007), 1-8.

[15] Ch. Kawan, On expanding maps and topological conjugacy, J. Difference Equ. Appl. 13 (2007), 803-820.

[16] J. Kobza, Iterative functional equations $x(x(t))=f(t)$ with $f(t)$ piecewise linear, J. Comput. Appl. Math. 115 (2000), 331-347.

[17] M. Kuczma, On the functional equation $\varphi^{n}(x)=g(x)$, Ann. Polon. Math. 11 (1961), 161-175.

[18] M. Kuczma, Functional Equations in a Single Variable, Monografie Mat. 46, Polish Scientific Publishers, Warszawa 1968.

[19] M. Kuczma, B. Choczewski and R. Ger, Iterative Functional Equations, Encyclopedia of Mathematics and Its Applications 32, Cambridge University Press, Cambridge 1990.

[20] M. Kuczma and A. Smajdor, Regular fractional iteration, Bull. Pol. Acad. Sci. Math. 19 (1971), 203-207.

[21] Zb. Leśniak, Constructions of fractional iterates of Sperner homeomorphisms of the plane, Iteration Theory (ECIT'92) (Batschuns), 182-192, World Scienific Publishing, River Rdge, New York, 1996.

[22] Zb. Leśniak, On fractional iterates of a homeomorphism of the plane, Ann. Polon. Math. 79 (2002), 129-137.

[23] Zb. Leśniak, On fractional iterates of a Brower homeomorphism embeddable in a flow, J. Math. Anal. Appl. 366 (2010), 310-318.

[24] Lin Li, J. Jarczyk, W. Jarczyk and Weinian Zhang, Iterative roots of mappings with a unique set-value point, Publ. Math. Debrecen 75 (2009), 203-220.

[25] Lin Li, Dilian Yang and Weinian Zhang, A note on iterative roots of PM functions, J. Math. Anal. Appl. 341 (2008), $1482-1486$.

[26] Liu Liu, W. Jarczyk, Lin Li and Weinian Zhang, Iterative roots of piecewise monotonic functions of nonmonotonicity height not less then 2, Nonlinear Anal. 75 (2012), 286-303.

[27] Liu Liu and Weinian Zhang, Non-monotonic iterative roots extended from characteristic intervals, J. Math. Anal. Appl. 378 (2011), 359-373.

[28] G. Łydzińska, On expanding iteration semigroups of set-valued functions, Math. Pannon. 15 (2004), 55-64.

[29] G. Łydzińska, On collapsing iteration semigroups of set-valued functions, Publ. Math. Debrecen. 64 (2004), 285-298.

[30] G. Łydzińska, Iteration families for which expansion implies collapse, Aequationes Math. 70 (2005), 247-253.

[31] G. Łydzińska, On lower semicontinuity of some set-valued iteration semigroups, Nonlinear Anal. 71 (2009), 5644-5654.

[32] Jie Hua Mai, Conditions for the existence of $N$ th iterative roots of self-homeomorphisms of the circle, Acta Math. Sinica 30 (1987), 280-283 (in Chinese).

[33] T. Powierża, Set-valued iterative square roots of bijections, Bull. Pol. Acad. Sci. Math. 47 (1999), 377-383.

[34] T. Powierża, Strong set-valued iterative roots, Grazer Math. Ber. 344 (2001), 51-56.

[35] T. Powierża, Higher order set-valued iterative roots of bijections, Publ. Math. Debrecen 61 (2002), 315-324.

[36] L. Reich, On power series transformations in one indeterminate having iterative roots of given order and with given multiplier, Iteration Theory (ECIT'91) (Lisbon), 210-216, World Scientific Publishing, River Edge, New York, 1992.

[37] L. Reich, On the local distribution of formal power series transformations in several variables with respect to embeddability. Iteration Theory (Batschuns, 1992), 236-244, World Sci. Publ. River Edge, NJ, 1996.

[38] L. Reich, On the distribution of formal power series transformations with respect to embeddability in the order topology, Österreich. Akad. Wiss. Math.-Natur. Kl. Sitzungsber. II 208 (1999), 97-114.

[39] L. Reich and J. Schwaiger, Über einen Satz von Shl. Sternberg in der Theorie der analytischen Iterationen, Monatsh. Math. 83 (1997), 207-221.

[40] L. Reich and J. Schwaiger, Linearisierung formal-biholomorpher Abbildungen und Iterationsprobleme, Aequationes Math. 20 (1980), 224-243.

[41] R. E. Rice, B. Schweizer and A. Sklar, When is $f(f(z))=a z^{2}+b z+c$ ?, Amer. Math. Monthly 87 (1980), 131-142.

[42] J. Schwaiger, Roots of formal power series in one variable, Aequationes Math. 29 (1985), 40-43.

[43] Yong-Guo Shi, Lin Li and Zb. Leśniak, On conjugacy of r-modal interval maps with non-monotonicity height equal to 1 , J. Difference Equ. Appl., DOI: 10.1080/10236198.2012.661727

[44] Yong-Guo Shi, Lin Li and Zb. Leśniak, Topological conjugacy of a class of r-modal interval maps (submitted).

[45] K. Simon, Some dual statements concerning Wiener measure and Baire category, Proc. Amer. Math. Soc. 106 (1989), 455-463.

[46] Ch.G. Small, Functional Equations and How to Solve Them, Problem Books in Mathematics, Springer, New York, 2007.

[47] Taixiang Sun, Iterative roots of anti- $N$-type functions on the interval, J. Math. Study 33 (2000), 274-280, 284 (in Chinese).

[48] Taixiang Sun and Hongjian Xi, Iterative roots of $N$-type functions on the interval, J. Math. Study 29 (1996), 40-45 (in Chinese).

[49] P. Solarz, On iterative roots of a homeomorphism of the circle with an irrational rotation number, Math. Pannon. 13 (2002), $137-145$.

[50] P. Solarz, On some iterative roots on the circle, Publ. Math. Debrecen 63 (2003), 677-692.

[51] P. Solarz, Iterative roots of some homeomorphisms with a rational rotation number, Aequationes Math. 72 (2006), $152-171$.

[52] P. Solarz, Iterative roots of homeomorphisms possessing periodic points, Ann. Acad. Pedagog. Crac. Stud. Math. 6 (2007), $77-93$. 
[53] P. Solarz, On some properties of orientation-preserving surjections on the circle, Math. Slovaca 57 (2007), $547-560$.

[54] P. Solarz, A note on some iterative roots, Ann. Univ. Pedagog. Crac. Stud. Math. 8 (2009), 57-66.

[55] P. Solarz, Some remarks on the general theorem of the existence of iterative roots of homeomorphisms with a rational rotation number, ESAIM: Proceedings 36 (2012), 26-31.

[56] P. Solarz, General theorem for the existence of iterative roots of homeomorphisms with periodic points, J. Math. Anal. Appl. 394 (2012), 391-399.

[57] Shl. Sternberg, Infinite Lie groups and formal aspects of dynamical systems, J. Math. Mech. 10 (1961), 451-474.

[58] Gy. Targonski, Topics in Iteration Theory, Vandenhoeck and Ruprecht, Göttingen 1981.

[59] Gy. Targonski, Phantom iterates of continuous functions, Iteration theory and its functional equations (Lochau, 1984), 196-202, Lecture Notes in Math., 1163, Springer, Berlin, 1985.

[60] Gy. Targonski, Progress of iteration theory since 1981, Aequationes Math. 50 (1995), 50-72.

[61] Bing Xu and Weinian Zhang, Construction of continuous solutions and stability for the polynomial-like iterative equation, J. Math. Anal. Appl. 325 (2007), 1160-1170.

[62] M.C. Zdun, On iterative roots of homeomorphisms of the circle, Bull. Polish Acad. Sci. Math. 48 (2000), $203-213$.

[63] M.C. Zdun, On set-valued iteration groups generated by commuting functions, J. Math. Anal. Appl. 398 (2013), 638-649.

[64] M.C. Zdun, On a limit formula for embedding of diffeomorphisms in regular iteration semigroups, J. Difference Equ. Appl. DOI:10.1080/10236198.2012.703661

[65] M.C. Zdun, The embedding problem in iteration theory (submitted).

[66] M.C. Zdun and P. Solarz, Recent results on iteration theory: Iteration groups and semigroups in the real case, Aequationes Math. DOI: $10.1007 /$ s00010-013-0186-x

[67] Guangyuan Zhang, Conjugation and iterative roots of a class of self-mappings of a line segment (I), Chinese Ann. Math. Ser A 13 (1992), 33-40 (in Chinese); Conjugation and iterative roots of a class of self-mappings of a line segment (II), ibid. 473-478 (in Chinese).

[68] Jingzhong Zhang and Lu Yang, Discussion on iterative roots of piecewise monotone functions, Acta Math. Sinica 26 (1983), 398-412 (in Chinese).

[69] Jingzhong Zhang, Lu Yang and Weinian Zhang Some advances on functional equations, Adv. Math. 24 (1995), $385-405$.

[70] Wanxiong Zhang and Weinian Zhang, Computing iterative roots of polygonal functions, J. Comput. Appl. Math. 205 (2007), 497-508.

[71] Weinian Zhang, A generic property of globally smooth iterative roots, Sci. China Ser. A 38 (1995), $267-272$.

[72] Weinian Zhang, PM functions, their characteristic intervals and iterative roots, Ann. Polon. Math. 65 (1997), $119-128$.

[73] Wenmeng Zhang, Yingying Zeng, W. Jarczyk, Weinian Zhang, Local $C^{1}$ stability versus global $C^{1}$ unstability for iterative roots, J. Math. Anal. Appl. 386 (2012), 75-82.

[74] Wenmeng Zhang and Weinian Zhang, Continuity of iteration and stability of iterative roots, J. Comput. Appl. Math. 235 (2011), 1232-1244

[75] G. Zimmermann, Über die Existenz Iterativer Würzeln von Abbildungen, Doctoral Dissertiation, Marburg/Lahn, 1978. 